\title{
Origin of Platelet-derived Growth Factor in Megakaryocytes in Guinea Pigs
}

\author{
arthur Chernoff, Richard F. Levine, and DeWitt S. Goodman, \\ Arteriosclerosis Research Center and the Department of Medicine, \\ Columbia University College of Physicians \& Surgeons, New York 10032; \\ Veterans Administration Medical Center, Washington, D. C. 20422
}

\begin{abstract}
A B S T RACT Growth factor activity, as determined by the stimulation of $\left[{ }^{3} \mathrm{H}\right]$ thymidine incorporation into the DNA of quiescent 3T3 cells in culture, was found in lysates of guinea pig platelets and megakaryocytes. Quantitative dilution studies demonstrated that, of the cells present in the guinea pig bone marrow, only the megakaryocyte possessed quantitatively significant growth factor activity. The amount of activity present in one megakaryocyte was equivalent to that present in 1,000-5,000 platelets, a value approximately comparable to the number of platelets shed from a single megakaryocyte. It is suggested that guinea pig platelet-derived growth factor has its origin in the megakaryocyte.
\end{abstract}

\section{INTRODUCTION}

Platelets contain a mitogen, termed the plateletderived growth factor (PDGF), ${ }^{1}$ which stimulates the proliferation of a variety of cell types in culture in vitro (1). Recent studies, with human platelets, have demonstrated that PDGF is localized in the platelet $\alpha$ granule (2-4), and that PDGF is secreted along with other $\alpha$-granule constituents after stimulation with thrombin, arachidonic acid, and collagen $(2,5)$. Interest in PDGF stems from its possible role in the pathogenesis of atherosclerosis $(6,7)$, and the more general

Received for publication 10 December 1979 and in revised form 21 January 1980.

1 Abbreviations used in this paper: FCS, fetal calf serum; PDGF, platelet-derived growth factor; PPP, platelet-poor plasma. role that it may play in regulating cell proliferation in concert with plasma-derived proteins.

No information is available about the origin of the PDGF found in circulating platelets. Three general possibilities exist. The first, that PDGF is synthesized in situ in platelets, is unlikely, because platelets have little protein synthetic capacity $(8,9)$. A second possibility is that PDGF is derived from outside the platelet, but then taken up by and concentrated within the platelet. A third, more likely possibility is that PDGF is synthesized in conjunction with $\alpha$ granule formation in the megakaryocyte. Megakaryocytes are known to be active in protein synthesis, and previous studies demonstrated that isolated guinea pig megakaryocytes, maintained in short-term culture, synthesize coagulation Factor VIII antigen, which is also found in guinea pig platelets (10). We now report studies on the presence and quantity of growth factor activity in purified guinea pig megakaryocytes that suggest that PDGF originates in that cell.

\section{METHODS}

Healthy Hartley strain guinea pigs were bled by cardiac puncture. Blood was drawn gently into a syringe containing one-ninth as much (by volume) $\mathrm{Na}$ citrate-citric acid solution, and was centrifuged at $1,200 \mathrm{~g}$ for $15 \mathrm{~min}$ at $20^{\circ} \mathrm{C}$ to obtain platelet-rich plasma. Platelet-rich plasma was centrifuged at $10,000 \mathrm{~g}$ for $30 \mathrm{~min}$ at $4^{\circ} \mathrm{C}$ to obtain a platelet pellet and platelet-poor plasma (PPP). The pelleted platelets were washed once by gentle suspension in phosphate-buffered saline, and were again pelleted and stored frozen at $-70^{\circ} \mathrm{C}$ until assayed.

Bone marrow cells were harvested from the femora and humeri of the same guinea pigs (above), into calcium- and 
magnesium-free Hanks' solution containing citrate, adenosine, and theophylline as described (11). Megakaryocytes were isolated from marrow cell suspensions by an albumin (bovine serum albumin, fraction V, Armour Pharmaceutical Co., Kankakee, Ill.) density gradient centrifugation, followed by two successive velocity sedimentations, as described $(10,11)$. The isolated megakaryocytes had been washed nine times by the completion of the separation procedures, and the other fractions six times. The cell fractions discarded after each step (the pellet in the density separations and the upper portion of the velocity sedimentations) contained modest numbers of megakaryocytes. These cell populations were saved for study, and are designated the waste cell fractions. The numbers of megakaryocytes, total cells, and platelets present were counted in each suspension by phase-contrast microscopy at $\times 250$. Megakaryocytes were identified by their size, nuclear configuration, and prominent light refraction.

Samples were prepared for growth factor assay by suspending the cells from the various pellets in phosphatebuffered saline, and then lysing the cells by freezing and thawing six times in dry ice-acetone. The lysates were heated at $56^{\circ} \mathrm{C}$ for $30 \mathrm{~min}$ and precipitated protein was removed by centrifugation. The supernates so obtained are referred to as lysates in this paper, and were assayed immediately after their preparation. PPP, when used in assays, was heat treated in a similar fashion. Protein content was estimated by the methods described $(12,13)$, and dilutions were made with phosphate-buffered saline.

Growth factor activity was assayed by measuring the stimulation of the incorporation of $\left[{ }^{3} \mathrm{H}\right]$ thymidine into DNA of Swiss albino mouse 3T3 cells (CCL 92, American Type Culture Collection, Rockville, Md.). The details of the cell culture techniques and of the protocol used for determining $\left[{ }^{3} \mathrm{H}\right]$ thymidine incorporation have been reported $(2)$. The experimental data were converted to arbitrary units of growth factor activity for convenience of quantitative expression, as described in detail elsewhere (4). In brief, the amount of growth factor activity in each test sample was measured by comparing it to the growth-prompting activity obtained with fetal calf serum (FCS, Gibco Laboratories, Grand Island Biological Co., Grand Island, N. Y.). To make such a comparison, a dose-response curve was determined in each experiment both for FCS and for each test sample. The doseresponse data were plotted semi-logarithmically, and one unit of growth factor activity was defined as the activity obtained with $1 \%$ FCS (in terms of the percentage of the activity obtained with $10 \%$ FCS). For each test sample, the amount of sample that exhibited $1 \mathrm{U}$ of growth factor activity (as determined from the FCS dose-response data) was determined from a semi-log plot of the dose-response data for the sample. In making these determinations, linear regression analyses were performed on those data comprising the linear portions of the semi-log plots. Finally, from the results obtained, the number of cells in a sample possessing $1 \mathrm{U}$ of growth factor activity was determined.

\section{RESULTS}

A preliminary experiment was conducted to determine whether guinea pig platelets possessed growth factor activity that was measurable in the bioassay developed for human PDGF. After lysis of unwashed platelets that were pelleted from platelet-rich plasma, distinct growth factor activity was observed, at a level of 10.4 $\mathrm{U} / 10^{9}$ platelets.
Experiments were next conducted to compare the growth factor activity of washed platelet lysates to that of megakaryocyte lysates (from different animals), with or without supplemental guinea pig PPP (2 mg protein/dish). In two experiments, in the presence of PPP, the platelet lysate activities were 10.2 and 16.2 $\mathrm{U} / 10^{9}$ platelets, respectively, and the corresponding megakaryocyte activities were 11.0 and $14.5 \mathrm{U} / 10^{6}$ megakaryocytes. Thus, under those conditions the isolated megakaryocytes contained distinct growth factor activity, comparable to that contained in $\sim 10^{3}$ times as many platelets.

Even in the absence of PPP, the level of growth factor activity derived from megakaryocytes was of the order of that derived from $10^{3}$ times as many platelets, although much lower growth factor activities were observed under these conditions. In the two experiments described above, in the absence of PPP, slightly $<1 \mathrm{U}$ of growth factor activity was observed per $10^{9}$ platelets or per $10^{6}$ megakaryocytes. These findings are similar to observations recently reported for human PDGF, that have demonstrated a synergistic interaction between PDGF and plasma-derived factors, that affects the amount of biological activity observed for a given amount of PDGF $(1,14,15){ }^{2}$

Megakaryocytes comprised $64 \%$ of the cell number and $\sim 98 \%$ of the mass of the cell pellets from which the megakaryocyte lysates were prepared $(10,11)$. It was possible, however, that a significant portion of the growth factor activity observed in the megakaryocyte lysates might have arisen from contaminating, nonmegakaryocyte cells. Accordingly, two additional experiments were conducted to compare lysates prepared from megakaryocytes to those from the waste cell fractions (i.e., the cells removed from the marrow cell suspensions during the various stages of megakaryocyte isolation). The results of these two experiments are shown in Figs. 1 and 2.

Fig. 1 shows the dose-response curves obtained with lysates prepared from purified megakaryocytes, from the first and second velocity waste cell fractions, and from washed platelets (from the same animals). Both the platelet and the megakaryocyte lysates showed a progressive increase in growth factor activity with an increase in the number of cells from which the amount of lysate tested had been obtained. By contrast, no such relationship was observed between growth factor activity and the cell number of the lysates from the first and second velocity waste fractions, and the growth factor activity of these fractions remained low, over the range of cell numbers tested (Fig. 1). Thus, of the narrow-derived cells, only the purified megakaryocyte

${ }^{2}$ We have found that the mitogenic activity of human platelet lysates is increased by as much as $10-15$-fold by adding $2 \%$ PPP serum to the incubation medium. Chernoff, A., and D. S. Goodman. Unpublished observation. 


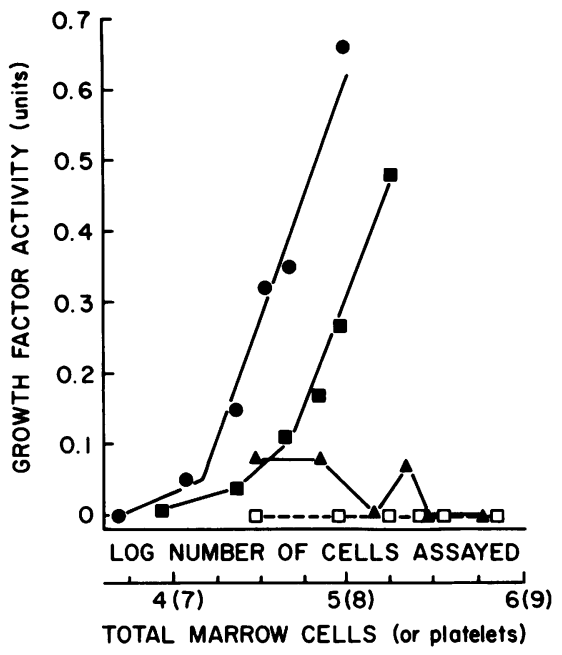

Figure 1 Growth factor dose-response curves for platelets and for different marrow cell fractions. The growth factor activities of increasing amounts of lysates obtained from megakaryocytes $(\boldsymbol{O})$, platelets $(\square)$, and from the first $(\square)$ and second $(\boldsymbol{\Delta})$ velocity waste cell fractions obtained during the megakaryocyte isolation procedure were determined. The results are plotted in terms of the total number of cells from which the amount of lysate assayed was derived. The total cell contents of the lysates assayed $\left(10^{6}\right.$ cells $/ \mathrm{ml}$ lysate $)$ and the percentage of cells as megakaryocytes were: isolated megakaryocytes $(0.48 ; 70 \%)$; second velocity waste $(2.83$; $1.8 \%)$; and first velocity waste $(3.47 ; 0.6 \%)$. Each point is the average of duplicate determinations.

fraction possessed considerable amounts of growth factor activity. Moreover, the dose-response curves obtained with the megakaryocyte and platelet lysates were roughly parallel to each other (Fig. 1). In this experiment, the potency of the purified megakaryocytes was $3.9 \mathrm{U}$ of growth factor activity per $10^{6}$ megakaryocytes, compared to $0.8 \mathrm{U} / 10^{9}$ platelets.

Fig. 2 shows the results of a second, similar experiment, in which the level of observed growth factor activity was related to the logarithm of the number of megakaryocytes present in each cell fraction. There was a high correlation $(r=0.82, P<0.001)$ between the growth factor activity observed in a given cell fraction and its megakaryocyte content. The specific activities (units $/ 10^{6}$ megakaryocytes) calculated for the various marrow fractions were all of the same order of magnitude (varying from 1.5 to $6.2 \mathrm{U} / 10^{6}$ megakaryo(ytes), despite the fact that the proportion of megakaryocytes (percentage of all marrow cells) varied almost three orders of magnitude in the different fractions. These data suggest that the growth factor activity present in guinea pig marrow is derived almost exclusively from the megakaryocytes.

Studies were also conducted to obtain information about the number of platelets derived from a single megakaryocyte. Previous estimates, based on extremely

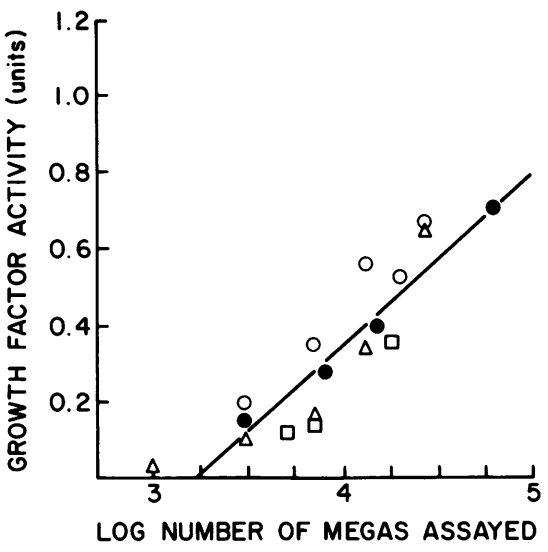

FIGURE 2 Growth factor dose-response curves for various marrow cell fractions, expressed as a function of the megakarayocyte content of each fraction. Lysates obtained from various fractions collected during the isolation of megakaryocytes were assayed at various concentrations. Isolated megakaryocytes $(\bullet)$, the first velocity waste cell fraction $(\square)$, the density waste cell fraction $(\triangle)$, and unfractionated bone marrow cells $(O)$ were assayed. The results are plotted in terms of the number of megakaryocytes (MEGAS) that were present in the sample from which the amount of lysate assayed was derived. The total cell contents of the lysates assayed $\left(10^{6}\right.$ cells $/ \mathrm{ml}$ lysate) and the percentage of cells as megakaryocytes were: isolated megakaryocytes $(0.39 ; 79 \%)$; first velocity waste $(5.52 ; 1.7 \%)$; density step waste $(78.9$; $0.2 \%)$; and marrow $(102 ; 0.3 \%)$. Each point is the average of duplicate determination.

limited data, have varied from about 2 to $11 \times 10^{3}(16)$. In one experiment, we determined the total protein content of pellets of isolated megakaryocytes and of washed platelets from the same guinea pigs. The total protein contents observed were $1.46 \mathrm{mg} / 10^{6}$ megakaryocytes and $1.45 \mu \mathrm{g}$ per $10^{6}$ platelets (means of two animals), representing a ratio of 1,000 platelets to one megakaryocyte in terms of total protein content. A second, morphometric study was also conducted to estimate the number of platelets in one megakaryocyte. From a fortuitous section of guinea pig bone marrow, an electron micrograph was made (17) of a fully mature megakaryocyte in which the cytoplasm was completely divided into platelets; shedding was probably imminent at the time of fixation. The size of this cell $(26 \times 42 \mu \mathrm{m})$ seems to be typical for a fully mature megakaryocyte. In one thin section, 233 discrete platelets were counted. Using $2.1 \mu \mathrm{m}$ as the thickness of these nondiscoid platelets, and Harker's calculation (18) for extrapolation from one section to the content of the entire ellipsoid, a total platelet count of 2,560 was estimated for this cell. This result is comparable to the protein content ratio (above), when one considers that the pool of isolated megakaryocytes comprised immature, developing, and fully mature cells, and that the latter alone would be expected to have a higher 
ratio of protein content (compared to platelets) than the isolated pool. Thus, the ratio of growth factor activities in megakaryocytes and platelets, which ranged from about 1,000 to 5,000, appears to be quantitatively similar to the number of platelets that are derived from a single megakaryocyte.

\section{DISCUSSION}

The 3T3 cell bioassay for growth factor activity has proven to be a useful quantitative assay for human PDGF under a variety of experimental circumstances $(2-5)$. As reported here, this sensitive bioassay is not stringently limited by problems of species specificity. Thus, the same bioassay developed for human PDGF could be used to detect and measure growth factor activity in the lysates of guinea pig platelets and megakaryocytes.

Human PDGF interacts synergistically with human $\operatorname{PPP}(1,14,15) .{ }^{2}$ Thus, it has been reported that maximal cell growth requires the presence both of platelet factors, which recruit quiescent cells into the cell cycle, and also of optimal amounts of plasma factors, which permit recruited cells to progress through the cell cycle $(14,15)$. The results reported here suggest that the growth factor activities present in both guinea pig platelets and megakaryocytes also demonstrate this biologic property of interacting synergistically with PPP.

The level of PDGF activity in guinea pig platelets, as measured in the bioassay used here, was approximately an order of magnitude less than that observed with human platelets, both in the absence and presence of PPP (4). At the present time we do not know if this difference is caused by the presence of different amounts of PDGF in guinea pig vs. human platelets, or to the possibility that PDGF from guinea pigs is much less active (for a given amount of PDGF mass) than human PDGF in this particular bioassay.

The aim of this study was to explore the question of the origin of PDGF. Previous studies have demonstrated the origin of two platelet components (Factor VIII antigen [10] and actin [19]) in the megakaryocyte. The evidence reported here supports the concept that guinea pig PDGF is present in guinea pig megakaryocytes, and hence presumably originates in the megakaryocyte. Although neither the identity of these guinea pig growth factors, nor the synthesis of growth factor by megakaryocytes has been established, the data do demonstrate the following points: (a) Guinea pig platelets possess growth factor activity that can be detected and quantified by a bioassay used for human PDGF. (b) Similarly, a preparation highly enriched in guinea pig megakaryocytes possesses growth factor activity. (c) Growth factor activity present in guinea pig marrow cells, or in the various waste cell fractions obtained during the process of megakaryocyte isolation, can be accounted for almost exclusively by the megakaryocyte content of each fraction. Thus, among cells present in bone marrow, only the megakaryocyte has significant growth factor activity. $(d)$ The number of platelets derived from a single megakaryocyte can be estimated to be of the order of $1-3 \times 10^{3}$, from measurements of the total protein contents of isolated megakaryocytes and washed platelets, and from morphometric analyses. (e) The growth factor activity present per megakaryocyte is sufficient to account for that found in $1-5 \times 10^{3}$ platelets. This value is comparable to the number of platelets that appear to be shed from a single megakaryocyte. Taken together, these findings indicate that PDGF is very likely to have its origin in the megakaryocyte.

\section{ACKNOWLEDGMENTS}

We are grateful to Ms. M. Polaneczky and Ms. K. Hazzard for their expert technical assistance.

This work was supported by National Institutes of Health grant HL 21006 (Specialized Center of Research), by The Veterans Administration, and by funds from The Schultz Foundation.

\section{REFERENCES}

1. Ross, R., and A. Vogel. 1978. The platelet-derived growth factor. Cell. 14: 203-210.

2. Witte. L. D., K. L. Kaplan, H. L. Nossel, B. A. Lages, H. J. Weiss, and D. S. Goodman. 1978. Studies of the release from human platelets of the growth factor for cultured human arterial smooth muscle cells. Circ. Res. 42: 402-409.

3. Kaplan, K. L., M. J. Broekman, A. Chernoff, G. R. Lesznik, and M. Drillings. 1979. Platelet $\alpha$-granule proteins: studies on release and subcellular localization. Blood. 53: 604-618.

4. Weiss, H. J., L. D. Witte, K. L. Kaplan, B. A. Lages, A. Chernoff, H. L. Nossel, D. S. Goodman, and H. R. Baumgartner. 1979. Heterogeneity in storage pool deficiency: studies on granule-bound substances in 18 patients including variants deficient in $\alpha$-granules, platelet factor 4 , $\beta$-thromboglobulin, and platelet-derived growth factor. Blood. 54: 1296-1319.

5. Linder, B. L., A. Chernoff, K. L. Kaplan, and D. S. Goodman. 1979. Release of platelet-derived growth factor from human platelets by arachidonic acid. Proc. Natl. Acad. Sci. U. S. A. 76: 4107-4111.

6. Ross, R., and J. A. Glomset. 1976. The pathogenesis of atherosclerosis. N. Engl. J. Med. 295: 369-377, 420-425.

7. Ross, R., and L. Harker. 1976. Hyperlipidemia and atherosclerosis. Chronic hyperlipidemia initiates and maintains lesions by endothelial cell desquamation and lipid accumulation. Science (Wash. D. C.). 193: 10941100.

8. Warshaw, A. L., L. Laster, and N. R. Shulman. 1967. Protein synthesis by human platelets. J. Biol. Chem. 242: 2094-2097.

9. Boullin, D.J., M. Votavova, and A. R. Green. 1972. Protein synthesis by human blood platelets after accumulation of leucine and arginine. Thromb. Diath. Haemorrh. 28: 54-64.

10. Nachman, R., R. Levine, and E. A. Jaffe. 1977. Synthesis 
of Factor VIII antigen by cultured guinea pig megakaryocytes. J. Clin. Invest. 60: 914-921.

11. Levine, R. F., and M. E. Fedorko. 1976. Isolation of intact megakaryocytes from guinea pig femoral marrow. Successful harvest made possible with inhibitors of platelet aggregation; enrichment achieved with a twostep separation technique. J. Cell Biol. 69: 159-172.

12. Waddell, W. J. 1956. A simple ultraviolet spectrophotometric method for the determination of protein. J. Lab. Clin. Med. 48: 311-314.

13. Murphy, J. B., and M. W. Kies. 1960. Note on spectrophotometric determination of proteins in dilute solutions. Biochim. Biophys. Acta. 45: 382-384.

14. Pledger, W. J., C. D. Stiles, H. N. Antoniades, and C. D. Scher. 1978. An ordered sequence of events is required before BALB/c-3T3 cells become committed to DNA synthesis. Proc. Natl. Acad. Sci. U. S. A. 75: 2839-2843.
15. Vogel, A., E. Raines, B. Kariya, M-J. Rivest, and R. Ross. 1978. Coordinate control of $3 \mathrm{~T} 3$ cell proliferation by platelet-derived growth factor and plasma components. Proc. Natl. Acad. Sci. U. S. A. 75: 2810-2814.

16. Kaufman, R. M., R. Airo, S. Pollack, and W. M. Crosby. 1965. Circulating megakaryocytes and platelet release in the lung. Blood. 26: 720-731.

17. Levine, R. F. 1977. Culture in vitro of isolated guinea pig megakaryocytes: recovery, survival, morphologic changes and maturation. Blood. 50: 713-725.

18. Harker, L. A. 1968. Megakaryocyte quantitation. J. Clin. Invest. 47: 452-457.

19. Nachman, R., R. Levine, and E. Jaffe. 1978. Synthesis of actin by cultured guinea pig megakaryocytes. Complex formation with fibrin. Biochim. Biophys. Acta. 543: 91105. 